\title{
Magnetic resonance imaging landmarks for preoperative localization of inferior medial genicular artery: a proof of concept analysis
}

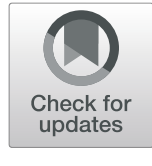

\author{
Ennio Sinno ${ }^{1 *}$, Armando Ugo Cavallo ${ }^{2,3}$, Gianluca Cera', Michele Dell'Orfano ${ }^{1}$, Daniele De Meo', \\ Massimiliano Sperandio ${ }^{3}$ and Ciro Villani ${ }^{1}$
}

\begin{abstract}
Introduction
Popliteal artery (PA) provides the blood supply to the anatomical components of the knee. At the lower limit of the popliteal fossa PA divides into anterior and posterior tibial arteries. The superior medial genicular artery, superior lateral genicular artery, inferior medial genicular artery (IMGA), inferior lateral genicular artery (ILGA) and middle genicular artery (MGA) are all collateral branches arising from PA. The anastomotic complex deriving from these vessels in addition to the descending geniculate artery, and the anterior and posterior recurrent arteries of the anterior tibial artery, supplies both soft tissues surrounding the knee, articular and osseous structures (Fig. 1) [2, 10].
\end{abstract}

\section{Anatomy of IMGA}

IMGA is one of the five collateral branches deriving from PA. Its origin can be superior, inferior or at the level of the joint line. From the popliteal fossa, the IMGA locates inferior to the medial condyle of the tibia, reaching the superficial medial collateral ligament [25]. Moreover, numerous anatomical variations had been reported in relation to its course. It could origin either directly as third, fourth or fifth branch from PA, or from a common trunk of PA, more frequently in combination with ILGA [23]. It supplies the medial proximal part of the tibia without any contribution from anastomotic vessels that supply the medial femoral condyle [12, 20, 27]. Then, it moves anteriorly to form an anastomotic complex with ILGA in the infrapatellar fat pad, thus

\footnotetext{
*Correspondence: enniosinno@gmail.com

'Department of Anatomical, Histological, Forensic Medicine and Orthopaedics Sciences, University "La Sapienza", Piazzale Aldo Moro, 5, 00185 Rome, Italy

Full list of author information is available at the end of the article
}

supplying the patella [11]. On its course, it provides cutaneous branches perforating at the lateral border of the sartorius muscle and at the medial border of the quadriceps tendon. Finally, it forms a common trunk with ILGA that initially branches to supply the anterior cruciate ligament $(\mathrm{ACL})$ and then travels through the intraarticular space to form a peri ligamentous anastomosis with the MGA, thus giving bloody supply to the postero-superior cruciate zone [17].

Numerous complications following its lesion after a variety of knee surgery procedures had been reported in the literature $[3,4,6-9,14-16,18,22,24]$.

Standard approaches that involve the medial aspect either of knee joint or of the tibial metaphysis, could determine a lesion to this vessel probably due to its numerous interindividual anatomical variations. On this basis, we retrospectively reviewed the anatomical course of IMGA on magnetic resonance imaging (MRI) scans in order to identify reproducible landmarks, which could be useful for preoperative planning and surgical procedures, that could help orthopedic surgeons to identify this vessel, thus to avoid any iatrogenic lesion.

\section{Methods \\ Patients \\ Magnetic Resonance images of the knee obtained with a high magnetic field MR scanner (Aera, 1.5 T, Siemens, Erlangen, Germany) were evaluated. Patients that under- went knee MRI due to unspecific knee pain were en- rolled. Eighty MRI from 80 patients (34 males and 46 females) were considered. The age of all patients was $50.7 \pm 15$ years $(44.8 \pm 17.6$ in males, $55 \pm 11.1$ in fe- males). All knee MRIs evaluated have been performed with standard protocols without gadolinium injection. Inclusion criteria were no previous knee and lower limb}



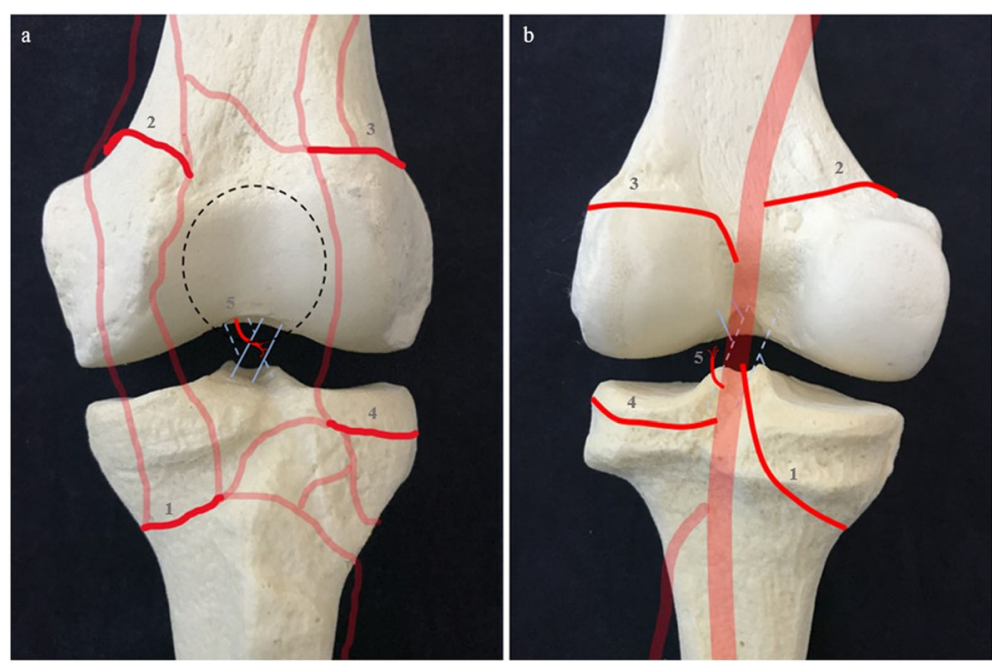

Fig. 1 Anterior (a) and posterior (b) courses of arterial vessels surrounding knee joint (in red) drawn on a 3D model. Legend: 1.Inferior medial genicular artery. 2.Superior medial genicular artery. 3.Superior lateral genicular artery. 4.Inferior lateral genicular artery. 5.Middle genicular artery. Black dotted line Patella. Light blue line Anterior cruciate ligament. Light blue dotted line Posterior cruciate ligament

vascular surgeries, arteriopathies and absence of arthritis deformity or direct trauma. Exclusion criteria were history of diabetes and smoking habits. Written informed consent was obtained by all patient.

\section{MRI technique and measurements}

The measurements were conducted on T2 weighted (T2w) with fat suppression sagittal (TR: $960 \mathrm{~ms}$; TE 23 ms; slice thickness $4 \mathrm{~mm}$ ) and coronal (TR: $3650 \mathrm{~ms}$; TE $38 \mathrm{~ms}$; TI $160 \mathrm{~ms}$; slice thickness $4 \mathrm{~mm}$ ) sequences. The MRI scan was conducted in supine position, with the knee in extension and the lower limb in neutral rotation.

Four parameters were considered for final analysis:

- distance from IMGA to the joint line in the sagittal view (IMGA-JL Sag) (Fig. 2),
- distance from IMGA to the joint line in the coronal view (IMGA-JL Cor) (Fig. 3),

- distance from IMGA to the border of the medial tibial plateau in the coronal view (IMGA-MTP Cor) (Fig. 4) and

- distance from IMGA to the insertion of semimembranous tendon on the posterior part of the medial tibial plateau in the coronal view (IMGASMT Cor) (Fig. 5).

The average value (IMGA-JL Avg) between IMGA-JL Sag and IMGA-JL Cor was also calculated.

All the measurements were performed twice by two orthopedic surgeons with a temporal interval of 6 weeks.

For sake of standardization a technical procedure was applied to each MRI scan: first, the identification of
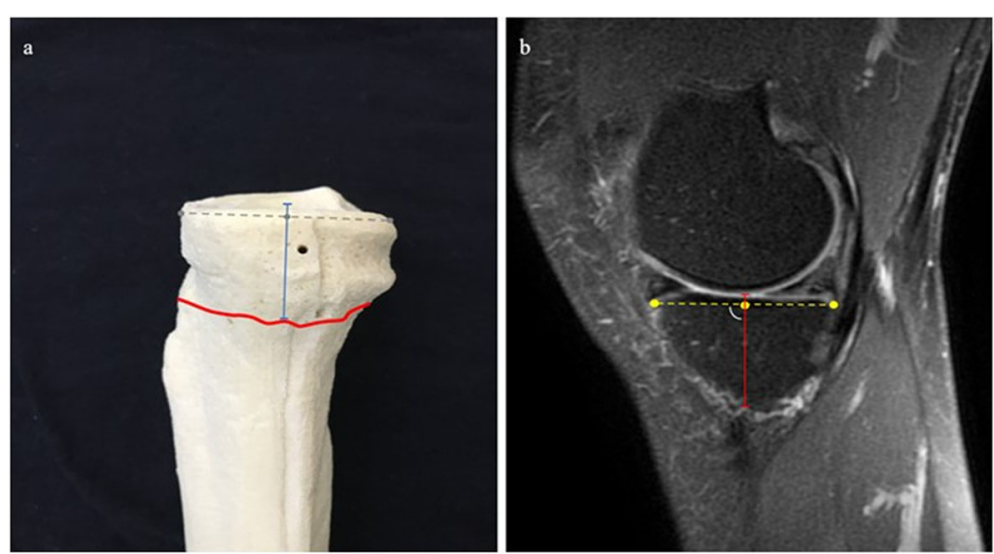

Fig. 2 a IMGA-JL Sag (blue) drawn on a 3D model. IMGA course in red and the reference line of the medial tibial plateau in grey. b. IMGA-JL Sag on T2w with fat suppression sagittal sequence (red) 

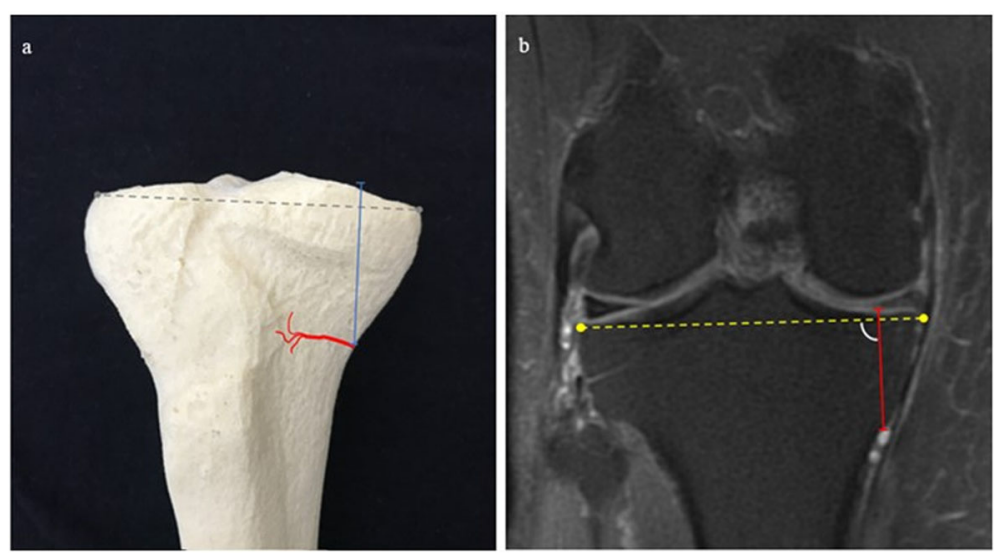

Fig. 3 a IMGA-JL Cor (blue) drawn on a 3D model. IMGA course in red and the reference line of the anterior tibial plateau in grey. b. IMGA-JL Cor on T2w with fat suppression coronal sequence (red)

IMGA on T2w sagittal and coronal sequences was evaluated; then, in T2w sagittal sequence, a line dividing the medial tibial plateau into two equal parts was drawn and the distance passing perpendicularly to that line, from IMGA to joint line (IMGA-JL Sag) was measured.

Then, on T2w coronal sequence, a line was drawn from the medial to the lateral border of the tibial plateau and the distance from IMGA to joint line (IMGA-JL Cor), was measured.

In the same sequence the distance from IMGA to the bony border of the tibial medial plateau (IMGA-JL Cor) was calculated. Finally, on T2w coronal sequence, the insertion of the semimembranosus tendon was identified on the posterior aspect of the medial tibial plateau and the distance from IMGA to it (IMGA-SMT Cor) was calculated.

\section{Statistical analysis}

Statistical analysis was performed with R V 3.4.4 (R Core Team (2018). R: A language and environment for statistical computing. R Foundation for Statistical Computing, Wien, Austria. URL https://www.r-project.org/). Continuous variables were analyzed with t-test or Wilcoxon test when appropriate. Inter- and intra-observer agreement was tested using the intraclass correlation coefficient (ICC). A $p$-value $<0.05$ was considered statistically significant.

\section{Results}

Considering all cases, IMGA-JL Sag was $30 \pm 4.3 \mathrm{~mm}$, IMGA-JL Cor was $29.9 \pm 4 \mathrm{~mm}$, IMGA-JL Avg was $29.9 \pm$ $4 \mathrm{~mm}$, IMGA-MTP Cor was $30 \pm 4.2 \mathrm{~mm}$ and IMGASMT Cor was $8.3 \pm 2.1 \mathrm{~mm}$ (Table 1 ). There was no statistically significant difference between IMGA-JL Sag and IMGA-JL Cor $(p=0.9)$. In males the value of IMGA-JL Sag was $32.7 \pm 3.8 \mathrm{~mm}$, in females was $28 \pm 3.5 \mathrm{~mm}(p<$ $0.001)$; the value of IMGA-JL Cor was $32.5 \pm 3.2 \mathrm{~mm}$ in males, $27.9 \pm 3.3 \mathrm{~mm}$ in females $(p<0.001)$. IMGA-JL Avg was $32.6 \pm 3.3 \mathrm{~mm}$ in males, $27.9 \pm 3.2 \mathrm{~mm}$ in females $(p<$ 0.001). IMGA-MTP Cor was $32.8 \pm 3.4 \mathrm{~mm}$ in males, $28 \pm$
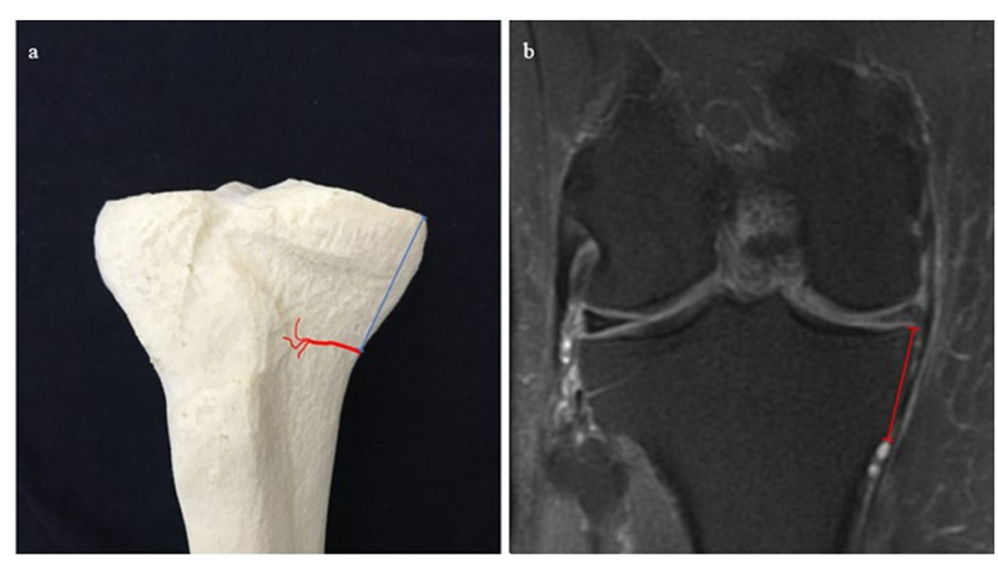

Fig. 4: a IMGA-MTP Cor (blue) drawn on a 3D model. IMGA course in red. b. IMGA-MTP Cor on T2w with fat suppression coronal sequence (red) 

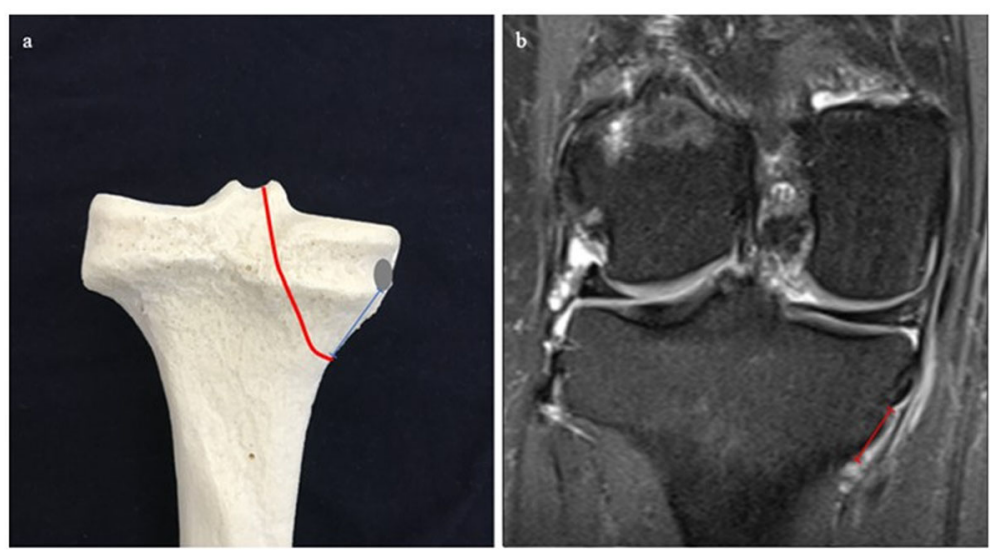

Fig. 5 a IMGA-SMT Cor (blue) drawn on a 3D model. IMGA course in red. Semimembranous tendon in grey. b. IMGA-SMT Cor on T2w with fat suppression coronal sequence (red)

$3.4 \mathrm{~mm}$ in females $(p<0.001)$. IMGA-SMT Cor was $8.7 \pm$ $2 \mathrm{~mm}$ in males, $8 \pm 2.2$ in females respectively $(p=0.1)$ (Table 2). The intraobserver agreement was 0.98 and interobserver agreement was 0.99 for the first measurement and 0.98 for the second measurement performed by both observers after 6 weeks.

\section{Discussion}

The most important finding in this study is that using MRI scans, routinely performed as preoperative instrumental investigation before knee surgery, it is possible to identify reliable reference points useful to avoid damage to IMGA during knee surgery. Furthermore it seems easy to recognize IMGA on MRI T2w with fat suppression sequences. With this approach, no further investigation, such as angio-CT or angiography could be needed to this purpose, resulting in cost-effectiveness for the patient. Moreover, these instrumental examinations, imply the infusion of contrast agents and contrast-specific techniques all having potential side effects as well as relative and absolute contraindications (allergic reaction, caution in case of patient affected by renal insufficiency, etc).

The correct application of the calculations shown above allows the localization of the IMGA course with reference to the joint line and the medial edge of the

Table 1 Baseline characteristics of patients included in the analysis

\begin{tabular}{ll}
\hline Age & $50.7 \pm 15$ \\
IMGA-JL Sag $(\mathrm{mm})$ & $30 \pm 4.3$ \\
IMGA-JL Cor $(\mathrm{mm})$ & $29.9 \pm 4$ \\
IMGA- JL Avg (mm) & $29.9 \pm 4$ \\
IMGA-MTP Cor $(\mathrm{mm})$ & $30 \pm 4.2$ \\
IMGA-SMT Cor $(\mathrm{mm})$ & $8.3 \pm 2.1$ \\
\hline
\end{tabular}

tibial plateau. The calculation of this distance allows the surgeon to act safely, avoiding lesions of this vessel. Using MRI it is also possible to measure the distance between IMGA and the tendon of the semimebranous, an anatomically important element in knee prosthetic surgery.

There are few studies that analyze the anatomic course of IMGA, either cadaveric or angiographic $[2,5,10,17,23]$.

To the best of our knowledge, it exists only one study based on MRI findings in relation to ILGA course [19]. Then, considering the risk of iatrogenic lesion of IMGA, it was attempted to assess its localization on MRI scans. All the measurements reported can be easily reproduced, as illustrated in the figures (Figs. 2, 3, 4 and 5). A useful landmark for surgical approaches to knee is the joint line. On this basis, the assessment of the distance between joint line and IMGA course could represent a valid guide for surgeons to avoid iatrogenic damage to this vessel. Being closely to real anatomy, this distance (IMGA-JL Avg) represents the mean value of the two measurements (IMGA-JL Sag and IMGA-JL Cor) drawn on MRI scans (Figs. 2 and 3). It was observed that IMGA-JL Avg had an interindividual variability, but considering the total amount of the male population, this length resulted greater $(32.6 \pm 3.3 \mathrm{~mm})$ than female

Table 2 Comparison between male and female patients

\begin{tabular}{llll}
\hline & M & F & $\boldsymbol{p}$-value \\
\hline Age & $44.85 \pm 17.57$ & $55 \pm 11.11$ & 0.005 \\
IMGA-JL Sag $(\mathrm{mm})$ & $32.66 \pm 3.8$ & $28.04 \pm 3.49$ & $<0.001$ \\
IMGA-JL Cor $(\mathrm{mm})$ & $32.57 \pm 3.23$ & $27.86 \pm 3.35$ & $<0.001$ \\
IMGA-JL Avg $(\mathrm{mm})$ & $32.61 \pm 3.32$ & $27.95 \pm 3.18$ & $<0.001$ \\
IMGA-MTP Cor $(\mathrm{mm})$ & $32.83 \pm 3.43$ & $27.98 \pm 3.45$ & $<0.001$ \\
IMGA-SMT Cor $(\mathrm{mm})$ & $8.75 \pm 2.02$ & $7.96 \pm 2.18$ & 0.1 \\
\hline
\end{tabular}


$(27.9 \pm 3.1 \mathrm{~mm})(p<0.001)$. This discrepancy could be associated to the well-known different physiognomic features between males and females. As a result, IMGA course could be closer to the joint line in females than in males, resulting in obvious surgical implications. Another measured distance (IMGA-MTP Cor) was drawn from IMGA to the border of tibial medial plateau: this bony point was chosen in order to provide a reproducible and useful landmark for surgeons. Finally, it was considered the distance between IMGA and the tibial insertion of the semimembranous tendon (IMGA-SMT Cor). Although extremely variable between patients, this distance could reveal a consistent practical usefulness for surgeons, specifically during TKA procedures. Whiteside described surgical tecnique for ligament balancing for TKA and focusing on medial compartment, he suggested that, in specific cases, it was necessary to extend the release up to the contracted semimembranous [26]. In these cases, surgeon should proceed with caution to preserve the integrity of IMGA. In our population the mean value of IMGA-SMT Cor was $8.2 \pm 2.1$ $\mathrm{mm}$, suggesting a not negligible relation between these structures, although there were not observed statistically significant difference in males and females.

All knee surgical procedures could cause various iatrogenic injuries of this vessel. Nevertheless, there are several papers reporting post-surgical complications due to IMGA lesion, the most frequent of which seems to be pseudoaneurysm [3, 6-9, 16, 18, 22, 24]. Indeed, Filho et al. reviewed 40 papers in literature obtaining that the arteries most affected were the PA (45.83\%) and the IMGA (20.83\%) [9]. Patients affected by pseudoaneurysm referred to clinicians mainly for pain (36.55\%) and pulsatile tumor (31.18\%).

Medial approaches to proximal tibia could determine an IMGA lesion, due to its relationship with anatomic structures of the medial compartment of the knee.

Focusing on IMGA injury after arthroscopic procedures, partial medial meniscectomy had been reported as a possible cause [7]. It is well known to knee surgeons that the resection of the posterior horn of the medial meniscus should be performed avoiding excessive tibial external rotation. This maneuver brings closer PA and IMGA to this medial meniscus part [4]. Moreover, solely standard medial arthroscopic portal could lead to IMGA lesion, regardless the procedure performed [14].

ACL surgery could cause IMGA injury. Evans et al. reported the first case of IMGA pseudoaneurysm following ACL reconstruction using bone patellar tendon bone autograft; vessel damage was probably caused by the surgical technique, particularly while performing the tibial tunnel when the tibial periosteum needs to be lifted [8]. Mello et al., reporting another case of IMGA pseudoaneurysm after ACL reconstruction using bone patellar tendon bone autograft, hypothesized that the damage might be due to the preparation of the intercondilar notch, performed to have a good view of its roof and posterior wall. This procedure usually provides the removal of the posterior cruciate ligament synovial membrane which is fed by IMGA [15]. Moreover, Milankov et al. described IMGA injury after ACL reconstruction using quadrupled hamstring tendon. In this case the reason of the vascular complication could had been the graft removal from the medial side of proximal tibia [16].

In ACL reconstruction surgery using hamstring tendon, IMGA lesion could compromise the success of the procedure as its terminal branches may influence the maturation of the graft [1].

Even during high tibial osteotomy (HTO) procedures IMGA is at risk of injury; Bisicchia et al. tried to assess the risk of vascular injuries both in closing and opening wedge HTOs, quantifying the distances between osteotomy cuts and arteries, using three-dimensional reconstruction: it was observed that IMGA could be more at risk during opening wedge HTOs [5].

Unicompartmental knee arthroplasty or TKA are more commonly performed through medial arthrotomy nearly the IMGA course; a considerable bleeding may occur during surgery, from inferomedial side of the joint capsule probably caused by IMGA injury. Cauterization of the soft tissues on this side is often necessary to allow surgeons to have a proper view of the area. Some authors reported their experience on IMGA pseudoaneurysm after TKA, suggesting a possible injury mechanism caused by the release of medial structures during ligament balancing techniques in patients suffering for knee osteoarthritis associated with varus deformity [26]. During this procedure, care must be taken by the surgeon to avoid any damage to IMGA, since its anatomical strictly connection with MCL, proximal tibial periosteum, pes anserinus insertion, posterior oblique ligament and semimembranous tendon $[6,18]$.

Finally, trauma surgery for tibial fractures may be involved in IMGA lesion too. Bennet et al. reported a case of IMGA false aneurysm after tibial intramedullary nailing revision surgery [3]. Either the removal of the prior proximal interlocking screw from the medial side of proximal tibia or the reinsertion of the new one, could have caused the lesion. Thus, the surgical treatment of fractures involving the medial tibial plateau, best managed by subperiosteal internal fixation, could endanger IMGA. However, reports of pseudoaneurysm formation without direct vessel wall injury have also been described $[9,13,21]$.

This study tries to emphasize the importance of IMGA and its collateral branches supply to the medial compartment of the knee. For this reason, a lesion of this vessel could determine surgical failures or insufficient view of the surgical area. Providing accurate landmarks to 
identify IMGA course in the medial compartment could represent a useful tool for surgeons in order to avoid its iatrogenic lesions.

Furthermore, to our knowledge there are no similar investigations in literature referring to MRI evaluation of IMGA course.

Several limitations can be listed:

- the measurements were conducted only on MRI scans, without any in vivo confirmation;

- the determination of accurate references was made by the Authors on the basis of anatomical landmarks detected on MRI scans;

- all patients enrolled in the study came from the same ethnic group. Because of the difference in anatomical features between groups, caution in required in applying our results to general population;

- the evaluations were conducted on a small sample of MRIs. An analysis based on a more consistent group would increase the statistical accuracy of the measurements.

\section{Conclusion}

The joint line is a useful landmark to identify IMGA course during knee surgery. The IMGA course is closer to the joint line and to the border of the medial tibial plateau in females than in males. Although the interindividual variability these results should be taken into account when performing all surgical procedures involving the medial aspect of the knee. Similar interindividual distances were observed between IMGA and semimembranosus tendon insertion regardless of gender. However, the proximity to this tendon should be considered especially during specific cases of ligamentous balancing in TKA procedures.

\section{Acknowledgements}

None.

\section{Authors' contributions}

All authors have made substantial contributions to draw this study, specifically: E. Sinno, M. Dell'Orfano, D. De Meo and C. Villani made conception and wrote the paper, C. Villani and M. Sperandio made design and critic revision. C. Villani and D. De Meo made the measurements. A. U. Cavallo and G. Cera made conception and statistical analysis. All the authors gave the final approval of submitted version.

\section{Funding}

No grant has been received for this study.

\section{Ethics approval and consent to participate} Not required.

\section{Consent for publication}

Acquired by all patients.

\section{Competing interests}

None.

\section{Author details}

${ }^{1}$ Department of Anatomical, Histological, Forensic Medicine and Orthopaedics Sciences, University "La Sapienza", Piazzale Aldo Moro, 5, 00185 Rome, Italy. ${ }^{2}$ Department of Biomedicine and Prevention, University "Tor Vergata", Rome, Italy. 'Division of Radiology, "San Carlo di Nancy Hospital", GVM Care and Research, Rome, Italy.

Received: 3 July 2020 Accepted: 10 September 2020

Published online: 28 September 2020

\section{References}

1. Arai Y, Hara K, Takahashi T, Urade H, Minami G, Takamiya H et al (2008) Evaluation of the vascular status of autogenous hamstring tendon grafts after anterior cruciate ligament reconstruction in humans using magnetic resonance angiography. Knee Surg Sports Traumatol Arthrosc 16(4):342-347

2. $\quad$ Barner KL, Mayer CM, Orth C, Tran QV, Olinger AB, Wright BW (2019) Mapping the Genicular arteries to provide a caution zone during knee surgery. Clin Anat 2019. https://doi.org/10.1002/ca.23535

3. Bennett FS, Born CT, Alexander J, Crincoli M (1994) False aneurysm of the medial inferior genicular artery after intramedullary nailing of the tibia. J Orthop Trauma 8:73-75

4. Bernard M, Grothues-Spork M, Georgoulis A, Hertel P (1994) Neural and vascular complications of arthroscopic meniscal surgery. Knee Surg Sports Traumatol Arthrosc 2(1):14-18

5. Bisicchia S, Rosso F, Pizzimenti MA, Rungprai C, Goetz JE, Amendola A (2015) Injury risk to extraosseous knee vasculature during osteotomies: a cadaveric study with $\mathrm{CT}$ and dissection analysis. Clin Orthop Relat Res 473(3):1030-1039

6. Dennis DA, Neumann RD, Toma P, Rosenberg G, Mallory TH (1987) Arteriovenous fistula with false aneurysm of the inferior medial geniculate artery. A complication of total knee arthroplasty. Clin Orthop Relat Res (222): $255-260$

7. Ellis CD (2016) Inferior medial geniculate artery pseudoaneurysm complicating arthroscopic partial meniscectomy. ANZ J Surg 88(7-8):E619E620

8. Evans JD, De Boer MT, Mayor P, Rees D, Guy AJ (2000) Pseudoaneurysm of the medial inferior genicular artery following anterior cruciate ligament reconstruction. Ann R Coll Surg Engl 82(3):182-184

9. Filho ES, Isolani GR, Baracho FR, De Oliveira Franco APG, Ridder Bauer LA, Namba M (2015) Pseudoaneurysm after arthroscopic procedure in the knee. Rev Bras Ortop 50(2):131-135

10. Hirtler L, Lübbers A, Rath C (2019) Vascular coverage of the anterior knee region - an anatomical study. J Anat 235(2):289-298

11. Jones KJ, Lazaro LE, Taylor SA, Pardee NC, Dyke JP, Hannafin JA et al (2016) Quantitative assessment of patellar vascularity following bone-patellar tendon-bone autograft harvest for $\mathrm{ACL}$ reconstruction. Knee Surg Sports Traumatol Arthrosc 24(9):2818-2824

12. Kirschner MH, Menck J, Hennerbichler A, Gaber O, Hofmann GO (1998) Importance of arterial blood supply to the femur and tibia for transplantation of vascularized femoral diaphyses and knee joints. World J Surg 22(8):845-851

13. La Perna L, Olin J, Goines D, Childs MB, Ouriel K (2000) Ultrasound-guided thrombin injection for the treatment of postcatheterization pseudoaneurysms. Circulation. 102:2391-2395

14. Lafontaine M, Khoury A (1997) False aneurysm of the knee after arthroscopy. Acta Orthop Belg 63(3):215-217

15. Mello W, de Brito WE, Migon EZ, Borges A (2011) Pseudoaneurysm of the medial inferior genicular artery after anterior cruciate ligament reconstruction. Arthroscopy. 27(3):442-445

16. Milankov M, Miljkovic N, Stankovic M (2006) Pseudoaneurysm of the medial inferior genicular artery following anterior cruciate ligament reconstruction with hamstring tendon autograft. Knee. 13(2):170-171

17. Nemschak G, Pretterklieber ML (2012) The patellar arterial supply via the Infrapatellar fat pad (of Hoffa): a combined anatomical and Angiographical analysis. Anat Res Int 2012:713838

18. Noorpuri BSW, Maxwell-Armstrong CA, Lamerton AJ (1999) Pseudoaneurysm of a geniculate collateral artery complicating total knee replacement. Eur J Vasc Endovasc Surg 18:534-535

19. Park J, Lee SS, Kim TW (2018) Quantitative analysis of the perimeniscal position of the inferior lateral genicular artery (ILGA): magnetic resonance imaging study. Surg Radiol Anat 40(7):823-828 
20. Reddy AS, Frederick RW (1998) Evaluation of the intraosseous and extraosseous blood supply to the distal femoral condyles. Am J Sports Med 26(3):415-419

21. Saini P, Meena S, Malhotra R, Gamanagatti S, Kumar V, Jain V (2013) Pseudoaneurysm of the superior lateral genicular artery: case report of a rare complication after total knee arthroplasty. Patient Saf Surg 7:1-4

22. Sarrosa EA, Ogilvie-Harris DJ (1997) Pseudoaneurysm as a complication of knee arthroscopy. Arthroscopy 13(5):644-645

23. Shahid S, Saghir N, Cawley O, Saujani S (2015) A cadaveric study of the branching pattern and diameter of the Genicular arteries: a focus on the middle Genicular artery. J Knee Surg 28(5):417-424

24. Sharma H, Singh GK, Cavanagh SP, Kay D (2006) Pseudoaneurysm of the inferior medial geniculate artery following primary total knee arthroplasty: delayed presentation with recurrent haemorrhagic episodes. Knee Surg Sports Traumatol Arthrosc 14:153-155

25. Toy BJ, Yeasting RA, Morse DE, McCann P (1995) Arterial supply to the human anterior cruciate ligament. J Athl Train 30:149-152

26. Whiteside LA (2004) Ligament balancing in Total knee Arthroplasty - an instructional manual, Springer

27. Yamamoto H, Jones DB Jr, Moran SL, Bishop AT, Shin AY (2010) The arterial anatomy of the medial femoral condyle and its clinical implications. J Hand Surg Eur 35(7):569-574

\section{Publisher's Note}

Springer Nature remains neutral with regard to jurisdictional claims in published maps and institutional affiliations.

\section{Submit your manuscript to a SpringerOpen ${ }^{\circ}$ journal and benefit from:}

- Convenient online submission

- Rigorous peer review

- Open access: articles freely available online

- High visibility within the field

Retaining the copyright to your article

Submit your next manuscript at $\boldsymbol{\nabla}$ springeropen.com 